Résumés des conférences et travaux

\title{
Histoire et civilisation du livre
}

\author{
Frédéric Barbier
}

\section{OpenEdition \\ Journals}

Édition électronique

URL : https://journals.openedition.org/ashp/1032

DOI : $10.4000 /$ ashp.1032

ISSN : 1969-6310

Éditeur

Publications de l'École Pratique des Hautes Études

\section{Édition imprimée}

Date de publication : 2 février 2011

Pagination : 266-275

ISSN : 0766-0677

\section{Référence électronique}

Frédéric Barbier, "Histoire et civilisation du livre », Annuaire de l'École pratique des hautes études (EPHE), Section des sciences historiques et philologiques [En ligne], 141 | 2011, mis en ligne le 24 février 2011 , consulté le 06 juillet 2021. URL : http://journals.openedition.org/ashp/1032 ; DOI : https://doi.org/ 10.4000/ashp.1032 


\title{
HISTOIRE ET CIVILISATION DU LIVRE
}

\author{
Directeur d'études : M. Frédéric BARBIER
}

Programme de l'année 2008-2009: Histoire du livre, des collections et des bibliothèques à l'époque moderne.

Après la parution de L'Europe de Gutenberg ${ }^{1}$, la conférence d'histoire et civilisation du livre avait plus particulièrement traité, en 2007-2008, du cas du Narrenschiff, la célèbre Nef des fous de Sébastien Brant. Elle a poursuivi dans la même direction en 2008-2009 : il s'agissait d'approfondir une problématique permettant de préciser les conditions dans lesquelles la «première révolution du livre »s'est développée et a progressivement impulsé des transformations de plus en plus profondes tant sur le plan des différents acteurs de la « librairie » (y compris le public des lecteurs), que sur celui de la mise en forme des livres et de l'évolution de leurs contenus.

\section{Socle théorique}

Le socle théorique principal sur lequel s'appuie la démonstration est donné par la typologie de l'innovation : la dialectique construite entre innovation de procédé (l'invention d'un procédé de fabrication nouveau, en l'espèce la typographie en caractères mobiles) et innovation de produit a d'abord retenu l'attention de la conférence. La présentation de cette problématique a fait l'objet, outre les conférences proprement dites de l'École pratique des hautes études, d'une intervention au CEFres de Prague ${ }^{2}$.

Dans un premier temps, l'imprimerie fonctionne en effet comme un système de reproduction, au sens propre du terme : il s'agit de reproduire ${ }^{3}$, sur le plan matériel, ce qui existe déjà, à savoir les manuscrits (1452-1455). C'est dans cette perspective que Gutenberg et ses premiers successeurs travaillent, et c'est cette perspective qui explique pourquoi ils ne tirent nullement toutes les conséquences de leur invention au niveau de la fabrication elle-même. La première phase de développement de l'imprimerie s'achève dans les années 1470, alors que les ateliers se multiplient dans les pays allemands et à l'étranger (Italie et France), et qu'une concurrence plus sévère commence à faire sentir ses effets.

Après l'innovation de procédé, la seconde phase sera donc marquée par l'innovation de produit, laquelle a été plus particulièrement envisagée par la conférence sous trois aspects différents :

1. Frédéric Barbier, L'Europe de Gutenberg. Le livre et l'invention de la modernité occidentale (XIII ${ }^{e}$ $X V I^{e}$ siècle), Paris, Belin, 2006 (Histoire et société), 364 p., ill., cartes et graph., couv. ill. en coul.

2. Conférence reprise et élargie à l'occasion du colloque de Rio de Janeiro en 2009.

3. La théorie de la reproduction a fait l'objet d'une conférence publique prononcée à Imola, et actuellement sous presse (« Reproduction du patrimoine livresque »). 
1) D'abord, l'apparition et le développement de l'édition en langue vulgaire. Cette question a fait notamment l'objet d'une contribution dans le cadre du recueil sur Les Langues imprimées publié en $2008^{1}$.

2) L'invention du livre imprimé illustré, en Allemagne à Bamberg et, en France, à Lyon : s'agissant de la France, ce sont en effet les imprimeurs et libraires lyonnais qui se trouvent plus particulièrement présents dans le domaine de l'innovation, dans la mesure où ils sont en concurrence avec un certain nombre d'autres villes, dont Paris, où le marché traditionnel de l'Université, de la cour, etc., est évidemment plus porteur. On sait que Lyon verra successivement la parution du premier livre en français, la Bible abrégée de $1473^{2}$, et celle du premier livre imprimé illustré en France, le Miroir de rédemption de l'humain lignaige donné par Martin Husz en $1478^{3}$. Ce dernier titre résulte d'un processus de transfert, dans la mesure où Husz, qui vient du Wurtemberg et a travaillé à Bâle, reprend pour son Miroir des bois allemands utilisés pour un Spieghel dès $1476^{4}$. L'ouvrage est adapté en français par le Lyonnais Julien Macho. À côté des conférences de l'EPHE, cet aspect de la problématique a été présenté à l'occasion d'une conférence publique tenue à l'université de Grenoble (« Le texte et l'image : quelques observations sur le livre imprimé à l'aube de la période moderne »), conférence actuellement sous presse.

3) Enfin, le troisième terme concerne l'évolution progressive de la « mise en livre », évolution qui conduit à l'invention du livre imprimé moderne en tant que quelque chose de radicalement différent du manuscrit. La conférence a plus particulièrement envisagé la question de l' « étiquette », autrement dit de l'invention de la page de titre.

Un aspect complémentaire, mais très important, de cette problématique a été traité de manière plus rapide : il s'agissait de préciser la manière dont les structures plus larges encadrant le « petit monde du livre » et le donnant à comprendre (notamment tout ce qui relève de l'infrastructure matérielle : technique, économie, etc.) s'articulaient avec un certain modèle de pratiques et de représentations, du côté tant des professionnels (typographes, libraires, de fonds et différents acteurs dans le domaine de la diffusion) que du public des lecteurs ${ }^{5}$. Cette approche met en œuvre un certain nombre de catégories relevant du champ de l'ethnographie et de l'anthropologie histo-

1. Frédéric Barbier, «L'invention de l'imprimerie et l'économie des langues en Europe au XV siècle », dans Frédéric Barbier (dir.), Les Langues imprimées (Histoire et civilisation du livre, 4), Genève, Droz, 2008 , p. 21-46, ill. Il est à noter que ce dossier a été prolongé par plusieurs articles publiés en 2009, à l'initiative du directeur d'études, et consacrés au problème des langues d'édition en Slovénie actuelle depuis le $\mathrm{XVI}^{\mathrm{e}}$ siècle, à la constitution d'une « librairie française » à Berlin après la Révocation de l'Édit de Nantes, aux pratiques de la langue dans la Bohème des Lumières et à l'édition en langue régionale dans le Nord de la France au début du Xx ${ }^{\mathrm{e}}$ siècle.

2. Pierre Aquilon, « La Bible abrégée », Revue française d'histoire du livre, 1972, p. 3-39.

3. Speculum humanæe salvationis [français], trad. Julien Macho, [Lyon, Martin Husz], 26 VIII 1478 (Goff, S 661).

4. Speculum humanae salvationis [allemand], Basel, Bernhard Richel, 31 VIII 1476 (HC 14936*). Cf. Lucien Febvre et Henri-Jean Martin, L’Apparition du livre, 3 éd., posf. de Frédéric Barbier, Paris, Albin Michel, 1999, p. 136.

5. À ce stade, la question des auteurs est réservée, mais elle a été abordée par : Frédéric Barbier, « Gutenberg et la naissance de l'auteur », Gutenberg Jahrbuch, 83 (2008), p. 109-127, ill. 
riques ${ }^{1}$ : les structures sont « habitées » par une des pratiques professionnelles qui leur permettent de fonctionner, et qui seront très progressivement codifiées (par exemple en ce qui concerne les pratiques d'apprentissage). Mais l'innovation touche aussi les pratiques de diffusion et de «consommation » des livres : après le temps des voyageurs itinérants et des représentants dans telle ou telle ville, la fin du $\mathrm{XV}^{\mathrm{e}}$ et le début du $\mathrm{XVI}^{\mathrm{e}}$ siècle survient le temps de la première mise en place des systèmes modernes de diffusion, à savoir les librairies. On sait que la première représentation connue d'une librairie est elle aussi d'origine lyonnaise : il s'agit de la célèbre Danse macabre des imprimeurs publiée par Husz dans les années 1500.

Ajoutons que, sur le plan méthodologique, le schéma général ainsi posé ne saurait se réduire de bonne foi à une simple étiquette d'un quelconque déterminisme technique : ce n'est pas l'objet (le livre), ni l'économie de sa production et de sa diffusion qui déterminent les choix des acteurs (de l'auteur au lecteur), leurs représentations, ni mêmes leurs pratiques. En revanche, les catégories de la technique (au sens large) et de l'économie tracent comme un horizon de choses possibles, de choses connues, d'habitudes et de modèles, par rapport auquel, de manière plus ou moins novatrice, se construisent les représentations des uns et des autres, se déterminent leurs choix et se déploient leurs pratiques. Les pratiques et les représentations du « petit monde du livre » et de la société plus large qui utilise le média de l'imprimé ne peuvent logiquement se donner à comprendre que par rapport aux conditions de fonctionnement de celui-ci.

\section{Problématique de l'étude de cas}

Dans un second temps, la conférence a cherché à mettre en œuvre cette problématique à partir de l'exemple du Compost et calendrier des bergers.

Pourquoi passer à l'étude précise d'un texte et des ses éditions successives? C'est que le troisième terme de l'innovation représentée par l'invention de l'imprimerie, puis du livre imprimé, concerne le public des lecteurs, et qu'il peut être brièvement présenté dans le cadre théorique d'une économie de la consommation. Avec la typographie en caractères mobiles, la production livresque (ou, plus largement, la production imprimée) augmente dans des proportions très importantes, et que l'on n'attendait certainement pas a priori - rappelons que l'on conserve aujourd'hui quelque trente mille titres incunables, ce qui peut correspondre à quinze millions d'unités bibliographiques mises en circulation en moins d'un demi-siècle. D'une part, cette véritable « révolution » implique, pour réussir, que les lecteurs traditionnels (les lecteurs de manuscrits) se procurent effectivement les nouveaux imprimés qui leur sont proposés, mais aussi que de nouveaux lecteurs interviennent progressivement en tant qu'acteurs sur le marché moderne du livre. L'innovation de procédé et l'innovation de produit sont nécessairement prolongées par l'innovation dans le domaine de la « consommation » des livres et autres objets imprimés (affiches et placards, plaquettes, etc.).

1. Méthodologie facilitant dans une certaine mesure le comparatisme chronologique. Voir, à titre d'exemple : Frédéric Barbier, « Pour une anthropologie culturelle des libraires : note sur la librairie savante à Paris au XIX ${ }^{\mathrm{e}}$ siècle », dans Jean-Yves Mollier (dir.), Une capitale internationale du livre : Paris, $X V I I^{e}-X X^{e}$ siècle (Histoire et civilisation du livre, 5), Genève, Droz, 2009, p. 101-120, ill. 
Ces phénomènes ont été analysés par la conférence en mettant en œuvre la catégorie de « retentissement » : selon quelles logiques et selon quels rythmes l'articulation se fait-elle entre un ensemble de transformations touchant le domaine des moyens sociaux de communication (des médias) et la société plus large? La catégorie du « retentissement » présente notamment le double avantage méthodologique d'induire d'entrée une certaine distance chronologique (déjà mise en évidence par l'analyse de typologie de l'innovation ${ }^{1}$ ) et de faciliter l'approche comparative (par exemple entre les différentes phases de mutation que l'on a désignées comme marquant les « révolutions » successives du livre, et notamment avec la révolution actuelle des « nouveaux médias » $)^{2}$. Ce retentissement se manifeste par le biais aussi bien des pratiques de lecture que de l'encadrement social de la « librairie » : par exemple, les effets potentiellement subversifs de la «première révolution du livre » ne sont appréhendés que progressivement, selon qu'ils se font sentir, et ce n'est qu'à la fin du $\mathrm{Xv}^{\mathrm{e}}$ et au début du $\mathrm{XVI}^{\mathrm{e}}$ siècle que se pose la question d'un éventuel encadrement des lectures et, le cas échéant, des activités liées au livre, au premier chef l'imprimerie. En France, la crise constituée par l' «Affaire des Placards », en 1534, illustre excellemment la thèse. Les sociétés occidentales sont les premières du monde à avoir été soumises aux effets d'un phénomène de médiatisation de masse et à mettre progressivement en place, selon des modalités d'ailleurs variables, les conditions nouvelles de fonctionnement de la branche qui caractériseront l'époque moderne, de la fin du $X^{\mathrm{e}}$ siècle au dernier tiers du XVIII ${ }^{\mathrm{e}}$ siècle. Par un certain nombre de mentions nouvelles relevant notamment du paratexte, le livre rend d'ailleurs lui-même compte de ces transformations (on pense par exemple aux mentions de privilège, etc.).

En revanche, tout ce qui touche à l'évolution des pratiques même de la lecture et des modes de « consommation » de ceux qui utilisent le média nouveau de l'imprimé pose un problème de sources qui a été maintes fois souligné par les chercheurs. Il est assez logique que l'historien du livre fasse de l'objet livre lui-même une source centrale de son travail : prolongeant la problématique esquissée par Henri-Jean Martin avec le concept de « mise en livre », la conférence a donc entrepris d'étudier précisément un livre emblématique du $\mathrm{XV}^{\mathrm{e}}$ siècle, le Compost et calendrier des bergers, pour en tirer un certain nombre d'observations concernant les professionnels à l'origine de la publication et les lecteurs de l'ouvrage. À ce stade, la théorie et l'historiographie de la réception ont fait l'objet d'une présentation systématique dans le cadre de la conférence, en liaison avec la rédaction de l'article « Réception » à paraitre dans le Dictionnaire international du livre ${ }^{3}$.

1. Mais il ne faut pas induire directement de la succession à la causalité.

2. Frédéric Barbier (dir.), Les Trois révolutions du livre : actes du colloque international de Lyon/Villeurbanne (1998) [nº spécial de la Revue française d'histoire du livre, 106-109, 2000], Genève, Droz, 2001, 343 p., ill., cartes, graph. .

3. Frédéric Barbier, « Réception », à paraître dans Dictionnaire encyclopédique du livre, Paris, Éd. du Cercle de la Librairie, troisième volume, lettres $\mathrm{N}$ à Z (sous presse). 


\section{Le Compost et Calendrier des bergers}

Publié pour la première fois à Paris par Guy Marchant en 1491, le Compost est connu par dix éditions incunables (huit parisiennes et deux genevoises). Marchant, dont nous ne savons que trop peu de choses, viendrait de la région sud de la Champagne (Langres) ou de Bourgogne (Mâcon), et il est surtout remarquable par l'attention qu'il consacre à l'innovation éditoriale. On sait par exemple qu'il donne en 1485 la première Danse macabre imprimée, dont on pense qu'elle est inspirée de la fresque du charnier des Innocents. Le succès du titre pousse Marchant à le faire traduire en latin par Pierre Desrey, de Troyes, et à le décliner à travers les différentes éditions de la Danse macabre des hommes, des femmes, etc. ${ }^{1}$ Le Compost et calendrier des bergers s'inscrit dans cette même logique de production associant des textes nouveaux à une mise en livre particulièrement soignée, souvent originale et dans laquelle une part importante est dévolue à l'illustration.

Le Compost et calendrier des bergers est un imprimé qui semble a priori paradoxal, en ce qu'il prétend transmettre une science «populaire » mais qu'il reste un volume destiné à une catégorie de lecteurs apparaissant comme privilégiés, voire comme très privilégiés - à commencer par le roi lui-même. L'objet est d'apporter par le livre une sorte de compendium de la science accumulée depuis les origines par cette figure mythique qu'est le berger : l'auteur du Compost se présente lui-même comme l'intermédiaire entre le monde des « bergers », qui n'ont « aucune cognoissane des escriptures, mais seulement par [leur] sens naturel et entendement », et celui des habitants des villes, des clercs et de tous ceux qui sont en mesure d'accéder au livre. La première légitimation du texte du Compost se fonde sur une référence de principe au savoir « naturel » qui est réputé être celui des bergers, mais cette articulation témoigne de ce que la référence est déjà, dans le même temps, largement étrangère ou extérieure au monde des lecteurs du volume.

De manière significative, la xylographie ouvrant le prologue représente l'auteur («Prologue de l'acteur », voir page suivante) : or, celui-ci n'est jamais nommé, son portrait n'est pas individualisé, et la figure de l'auteur comme intermédiaire entre le monde des bergers et celui des lecteurs relève de la figure de style plus que de la réalité. L'étude du texte démontre que celui-ci constitue un recueil composite probablement organisé à l'initiative et dans l'atelier même de Marchant, sur la MontagneSainte-Geneviève. L'essor de l'économie moderne du livre fait que, y compris pour ce qui relève de l'écriture elle-même, l'initiative passe désormais parfois aux mains du libraire, parfois qualifié de promotor (par exemple pour Johann Bergmann à Bâle) : il est très probable que Guy Marchant, s'il n'a pas rédigé lui-même, a mis à la disposition de ses rédacteurs un certain nombre de textes déjà publiés et dont ils se sont inspirés pour leur propre travail.

Le Compost met en œuvre une forme de connaissance traditionnelle, fondée sur les correspondances et sur les équivalences : les phénomènes du monde extérieur (le macrocosme) sont en liaison directe avec ceux du monde intérieur de chacun (le

1. Frédéric Barbier (dir.), Paris, capitale des livres. Le monde des livres et de la presse à Paris, du Moyen Âge au XX $X^{e}$ siècle, Paris, Paris Bibliothèques, Presses universitaires de France, 2007, notamment p. 8889. Une édition parisienne du Compost est également présentée, p. 90-91. 


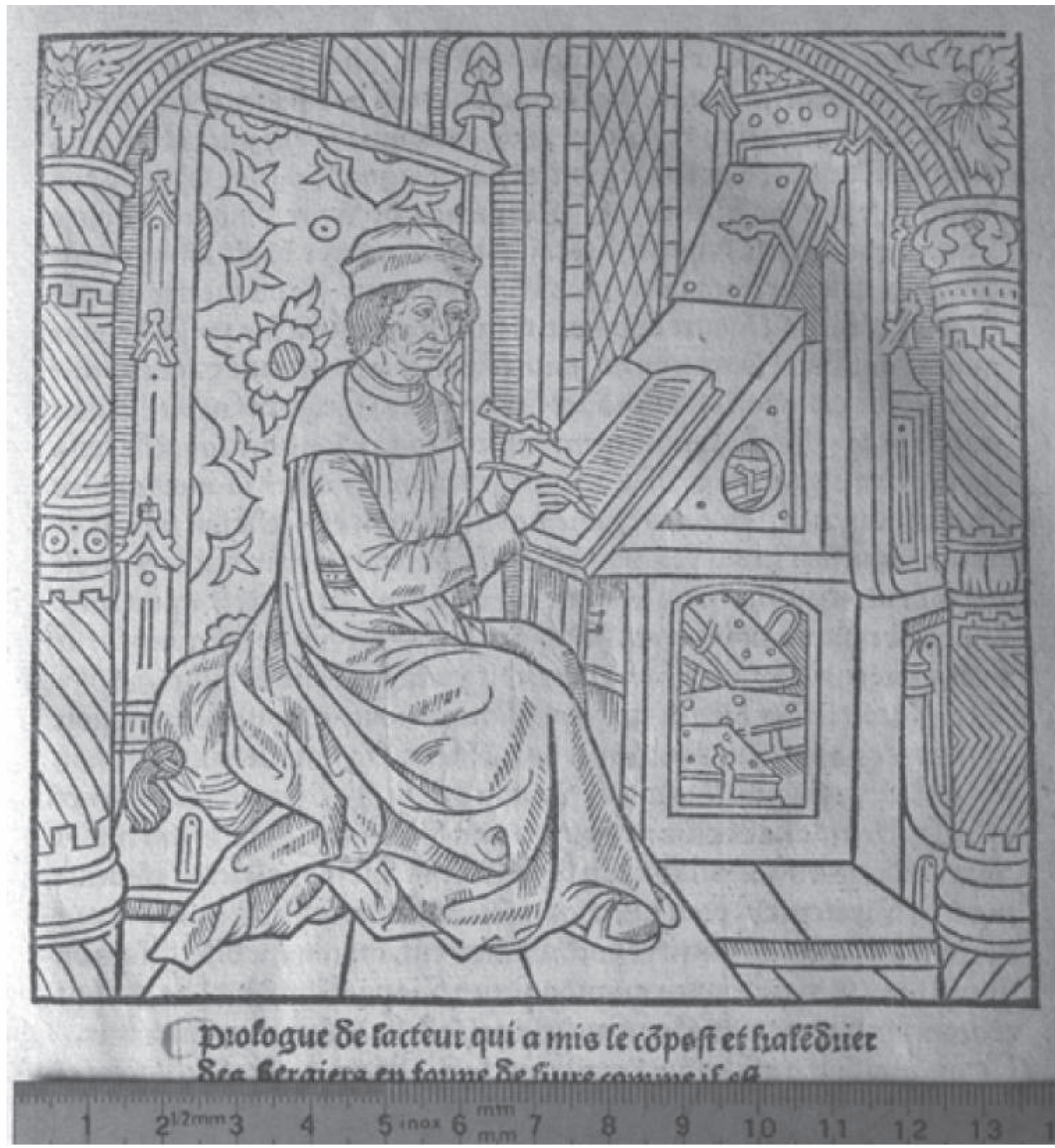

microcosme), de sorte que le mouvement des astres, la succession des saisons, etc., déterminent certains aspects de la vie individuelle, surtout s'agissant de la santé, mais aussi des prédictions, etc. Bien entendu, le contenu de l'ouvrage offre aussi une dimension morale très présente, spectaculairement mise en scène par une succession de très belles gravures. Contrairement à la tradition savante, qui voit dans le Compost le modèle de l'almanach ${ }^{1}$, il s'agit plutôt d'un compendium encyclopédique censé faciliter à chacun la conduite de sa vie quotidienne.

1. Dictionnaire encyclopédique du livre, I, p. 63-64. Sur la littérature des almanachs, voir notamment : Geneviève Bollème, «Littérature populaire et littérature de colportage au XVIII ${ }^{\mathrm{e}}$ siècle », dans Livre et société en France au XVIII ${ }^{e}$ siècle, Paris, 1965, p. 61-92; Id., Les Almanachs populaires aux XVII et XVIII siècles. Essai d'histoire sociale, Paris - La Haye, Mouton, 1969; Id., La Bibliothèque bleue, Paris, 1971; Robert Mandrou, De la culture populaire aux XVII et XVIII siècles. La Bibliothèque

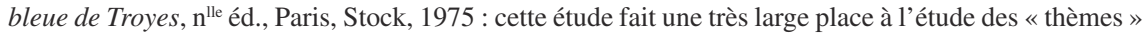
récurrents de la «Bibliothèque bleue », dans une optique relativement proche de celle de l'histoire littéraire. Sur les almanachs, le répertoire de base est toujours celui de John Grand-Carteret, Les Almanachs français. Bibliographie des almanachs..., Paris, 1896. Sur la « Bibliothèque bleue », cf. Alfred Morin, Catalogue descriptif de la Bibliothèque bleue de Troyes (almanachs exclus), Genève, Droz, 1974. 
L'étude de la mise en livre et celle des exemplaires conservés fournissent indirectement un certain nombre d'indications sur le lectorat potentiel à partir de la fin du $\mathrm{XV}^{\mathrm{e}}$ siècle. La mise en page est extrêmement soignée, la scansion est marquée par une ponctuation attentive (avec présence de majuscules), par le jeu des titres et sous-titres, et par la rubrication des pieds de mouche, tandis que le texte (en langue vulgaire) présente peu d'abréviations : c'est que le public visé n'est pas composé de clercs. L'organisation du texte est annoncée par le discours lui-même : il n'y a ni foliotation, ni tables, et nous restons dans une géométrie à deux dimensions, dont la mesure est constituée par la pagina. En revanche, il faut souligner la complexité de certains systèmes de codes, notamment les tableaux croisés utilisant une double couleur (noir/ rouge), et les lettres de l'homme phlébotomique renvoyant à la légende. De sorte que l'on peut bien penser le Compost en tant que un recueil est destiné à un lectorat de non-clercs, mais relativement instruit et possédant des moyens financiers non négligeables.

Nous sommes ainsi devant un modèle radicalement différent de celui de la lecture dite «populaire » auquel on réduit trop souvent le Compost et calendrier. Les considérations de Claudin et de Monceaux sont anachroniques : la Danse macabre de 1485 n'est évidemment pas une publication « qui s'adresse aux masses » (Claudin), ni le Compost « un almanach illustré à l'usage des gens de la campagne », voire un « ouvrage qui s'adressait aux masses populaires et rurales » (Monceaux). Cependant, il fonde une tradition, qui se développe dans l'espace de la librairie française : le Compost est effectivement organisé autour du calendrier, mais, contrairement au modèle germanique du Kalender, il y ajoute un ensemble de pièces à vocation pratique, relatives à la piété (d'une certaine manière, nous nous rapprochons du modèle du livre d'Heures), à l'horoscope (que faire, quand le faire et dans quelles conditions ?) ou à l'hygiène et à la santé.

Le succès même du Compost explique qu'il ait été régulièrement réédité durant tout l'Ancien Régime, toujours en présentant des textes qui reproduisent ceux du $\mathrm{XV}^{\mathrm{e}}$ siècle ou qui en sont extrêmement proches : une quarantaine d'éditions est connue jusqu'à la fin du XVIII ${ }^{e}$ siècle. Certaines sont données à Rouen, tandis que les années 1780-1800 voient l'extension du modèle à l'Alsace, avec notamment Colmar - nous laissons de côté les traductions en anglais et en allemand. La conférence a consacré la dernière partie de l'année à l'examen de ces éditions successives.

\section{La conjoncture $d u$ «populaire » et du «lettré »}

Henri-Jean Martin avait le premier envisagé le double rapport oral/écrit, savant/ populaire dans un article qui a fait date publié dans le Journal des savants en $1975^{1}$. Dans la prolongation de ces travaux, l'hypothèse que la conférence a explorée, en s'appuyant sur l'exemple du Compost, a consisté à articuler la conjoncture de la réception du texte à l'époque de la « librairie d'Ancien Régime » avec la rupture qui se produit au $\mathrm{XVII}^{\mathrm{e}}$ siècle dans l'ordre de la pensée scientifique : trois périodes se succèderaient dans cette trajectoire de notre texte.

1. Henri-Jean Martin, « Culture écrite et culture orale, culture savante et culture populaire dans la France d'ancien régime », Journal des savants, 1975, p. 225-282. 
1) Le Compost, succès parisien, est d'abord repris par un certain nombre de libraires de province et de l'étranger : Genève, Lyon, Rouen, etc., mais surtout Troyes (à partir de 1529). Il s'agit d'exploiter le titre en visant un public un peu plus large que celui touché par la seule librairie parisienne, et notamment le public aisé d'un certain nombre d'autres villes que la capitale. L'édition troyenne de 1529 , tout comme celle de Lyon en 1597, constituent ainsi des petits volumes recherchés, dont le style iconographique se renouvelle en se modernisant. Dans le même temps cependant, le paysage intellectuel change progressivement, de sorte le savoir traditionnel véhiculé par le Compost apparaît comme de plus en plus dépassé (il n'est plus pertinent), voire comme condamnable : comme le texte ne fait pas l'objet d'une mise à jour, il est déclassé aux yeux des prescripteurs.

2) On comprend dès lors comment le Compost entre, au XVII ${ }^{\mathrm{e}}$ siècle, dans la «Bibliothèque bleue », et comment il devient une des pièces de la littérature de colportage. On sait que la «Bibliothèque bleue » ne constitue pas une collection en soi, mais qu'il s'agit d'un terme générique désignant un certain type de produits imprimés, lesquels sont souvent vendus sous un brochage de couleur bleue ${ }^{1}$. La formule mise au point à Troyes par les imprimeurs et libraires Oudot (Nicolas [I], † 1636, et Nicolas [II], $\dagger 1692$ ) vise à répondre au marasme qui caractérise la librairie du royaume au début du siècle : elle consiste à exploiter un large fonds de textes et de bois gravés, en les réimprimant sur du papier médiocre et avec des caractères usés. Ces éditions dites «populaires » sont caractérisées par la référence à des textes souvent anciens (des « romans » médiévaux, des vies de saints, etc.), et, sur le plan formel, par l'emploi de bois, alors que la librairie parisienne est passée à la nouveauté et à la taille-douce. L'objectif est de diffuser le plus largement, à bon marché, en ville (les Troyens ont même des accords avec les libraires parisiens), mais aussi, de plus en plus à partir du milieu du $\mathrm{XVII}^{\mathrm{e}}$ siècle, en milieu rural et par le biais du colportage.

Ce qui définit le caractère plus ou moins « populaire » d'un imprimé, c'est donc moins son contenu en lui-même, que : 1) la déqualification de ce contenu; 2) la forme matérielle du volume; 3 ) son canal plus ou moins spécifique de distribution et notamment le colportage, canal dont l'administration se défie comme étant potentiellement dangereux; 4) enfin, bien évidemment, son prix de vente (Mandrou indique un prix de 1 à 2 sous par brochure).

3) Un nouveau renversement se produit dès lors que l'on rencontrera des intellectuels qui entreprennent de noter les textes des chansons ou des histoires, etc., interprétées devant eux. Le fait se produit occasionnellement dès le XVII ${ }^{\mathrm{e}}$ siècle - et il faut dès lors prendre garde à l'erreur d'interprétation, tel recueil « populaire » relevant au contraire d'une démarche éditoriale « distinguée », voire savante. La publication des Histoires, ou Contes du temps passé, avec des moralitez ${ }^{2}$ de Charles Perrault (1697)

1. Alfred Morin indique la date de 1657 comme étant celle de la première édition troyenne du Compost diffusée par colportage.

2. Charles Perrault, Histoires, ou Contes du temps passé, avec des moralitez, Paris, chez C. Barbin, 1697 , 273 p., 12 $2^{\circ}$. Le frontispice d'Antoine Clouzier est intitulé : «Contes de ma mère l'oye ». Les contes sont les suivants : La Belle au bois dormant; Le Petit Chaperon rouge; La Barbe bleue; Le Maistre chat, ou le Chat botté; Les Fées; Cendrillon, ou la Petite pantoufle de verre; Riquet à la houppe; Le Petit Poucet. L'ouvrage est dédié à « Mademoiselle », nièce de Louis XIV. Brunet IV, 507; Tchémerzine, 
s'insère dans une conjoncture intellectuelle spécifique, et inaugure en France un mouvement qui se prolonge surtout en Allemagne sous forme de réappropriations savantes ou littéraires : ce sera les travaux des frères Grimm, à terme (début du XIX ${ }^{\mathrm{e}}$ siècle) ceux du groupe de Heidelberg (Des Knaben Wunderhorn). On sait que Goethe réanime la tradition de Faust à partir d'un succédané du Volksbuch publié en prose à Francfort en 1587, puis en vers à Tübingen en 1587-1588. En 1783 est lancée une « Nouvelle bibliothèque bleue », réécrite, et dont le projet est de spéculer sur la curiosité d'un public différent pour les « romans » anciens :

Il paraîtra sans doute bien singulier qu'on ait pris la peine de rajeunir des ouvrages qui depuis plus de deux siècles sont abandonnés au peuple; des romans que la plus mince bourgeoise n'oserait se vanter d'avoir lus, non pas à cause du style et du langage (...) mais précisément parce qu'ils ont fait l'amusement de la plus vile populace (...).

Madame de N... sonna sa femme de chambre et lui demanda l'Histoire de Pierre de Provence. La soubrette étonnée se fit répéter jusqu'à trois fois, et reçut avec dédain cet ordre bizarre : il fallut pourtant obéir : elle descendit à la cuisine, et rapporta la brochure en rougissant... 1

Le XIX ${ }^{e}$ siècle sera d'abord marqué par la poursuite de cet inventaire d'un monde dont on pressent la disparition, y compris sur le plan linguistique (les patois et les dialectes) - et le travail de recensement réalisé par un Nisard se donne aussi à comprendre par rapport à cette conjoncture. Rudolf Schenda pense que le système ancien des savoirs, des apprentissages et des patrimoines culturels fondé sur l'oralité a été définitivement détruit dans les sociétés occidentales auX XIX ${ }^{\mathrm{e}}$ et $\mathrm{XX}^{\mathrm{e}}$ siècles : par suite, ses supports et ses produits sont décrits comme «populaires », c'est-à-dire comme implicitement non pertinents et pratiquement oubliés (les patois, les chants et récits, les contes, etc.).

Mais le XIX ${ }^{\mathrm{e}}$ siècle est aussi marqué par une politique systématique de destruction d'une « librairie populaire » considérée comme néfaste (parce qu'elle diffuse des modèles et des connaissances généralement périmés) et éventuellement comme dangereuse sur le plan politique. La succession en sera prise surtout par la presse périodique à grand tirage. De cette trajectoire complexe, le Compost et calendrier des bergers donne une illustration à la fois précise et très pertinente. Sa réédition récente sous forme de fac-similé savant ${ }^{2}$ couronne la transition d'un texte (et d'un livre) passé du statut de texte recherché et distingué à celui de texte populaire plus ou moins déclassé,

V, 177. Très peu d'exemplaires sont mentionnés par le CCF : BNF (2 ex.), Sorbonne, bibl. municipale de Marseille. Une contrefaçon est donnée cette même année (BNF). La bibliothèque municipale de Toulouse possède un exemplaire d'une contrefaçon qualifiée de « brochure de colportage », et il existe aussi une édition réalisée à Dôle en 1700 .

1. Histoire de Robert le Diable, duc de Normandie, éd. Jean Castilhon, à Paris, chez Fournier, libraire, rue du Hurepoix, près du pont Saint-Michel, à la Providence, 1783, $8^{\circ}$ (« La Bibliothèque bleue, entièrement refondue \& considérablement augmentée », 2). On remarquera que cette édition présente un frontispice gravé sur cuivre, ce qui ne correspond absolument pas à la mise en livre traditionnelle de la «Bibliothèque bleue » de Troyes : le lectorat visé n'est plus le même.

2. Calendrier des bergers, préface de Max Engammare, Genève, Paris, 2008, et compte rendu par Frédéric Barbier dans Histoire et civilisation du livre. Revue internationale, 5 (2009), p. 378-382. 
avant d'être réinvesti par le programme de la librairie savante et par les développements de problématique ethnographique moderne.

Grâce à l'obligeance du directeur et des conservateurs de la bibliothèque municipale du Mans (médiathèque Louis Aragon), la traditionnelle séance foraine organisée par la conférence a pu se dérouler dans cet établissement. La matinée a été consacrée à la présentation d'ouvrages manuscrits par $\mathbf{M}^{\text {me }}$ Geneviève Hasenohr et par M. Jean Vézin, tous deux correspondants de l'Institut; l'après-midi, à la présentation de quelques imprimés anciens commentés par MM. Pierre Aquilon (CESR de Tours), Frédéric Barbier et Didier Travier (conservateur chargé des fonds patrimoniaux à la bibliothèque du Mans). On a notamment pu étudier deux titres sortis de l'atelier de Guy Marchant : Ars moriendi (1483), et Compost et calendrier des bergers (1500); une Danse macabre troyenne de Nicolas Le Rouge; une rarissime édition nantaise des Lunettes des princes de Meschinot (1493); plusieurs éditions réalisées pour l'Église du Mans (Missel de 1483, Bréviaire de 1492, Manuale de 1505); enfin, quelques exemplaires provenant de la bibliothèque de Simon Hayeneufve et caractéristiques des développements de la Renaissance au Mans dans les premières décennies du XVI ${ }^{\mathrm{e}}$ siècle. 